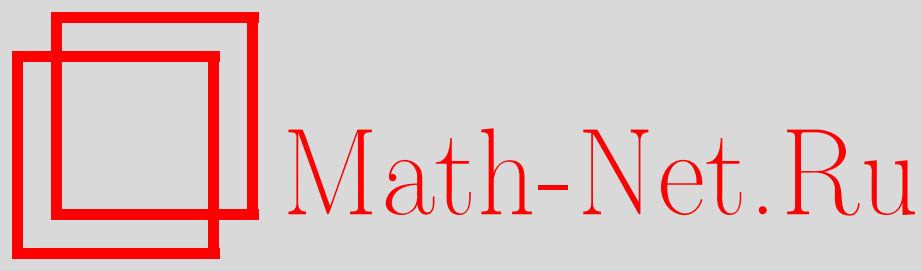

М. И. Тихомирова, В. П. Чистяков, Асимптотическая нормальность чисел непоявившихся значений $m$ зависимых случайных величин, Дискрет. матем., 2014, том 26, выпуск 2, 143-158

DOI: https://doi.org/10.4213/dm1285

Использование Общероссийского математического портала Math-Net.Ru подразумевает, что вы прочитали и согласны с пользовательским соглашением http: //www . mathnet.ru/rus/agreement

Параметры загрузки:

IP : 54.172 .240 .79

26 апреля 2023 г., 13:47:55

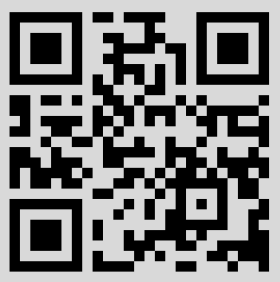




\section{Асимптотическая нормальность чисел непоявившихся значений $m$-зависимых случайных величин}

() 2014 г. М. И. Тихомирова*, В. П. Чистяков ${ }^{\dagger}$

Рассматриваются числа непоявившихся значений в отрезках стационарной последовательности $m$-зависимых случайных величин с $N$ исходами. Доказана совместная асимптотическая нормальность этих чисел при условии, что число $N$ и длины отрезков стремятся к бесконечности и имеют одинаковый порядок роста.

Ключевые слова: $m$-зависимые случайные величины, число непоявившихся значений.

Пусть $X=\left(x_{1}, x_{2}, \ldots, x_{t}, \ldots\right)$ - стационарная последовательность случайных величин, принимающих значения из множества $\aleph=\{1,2, \ldots, N\}$. Обозначим через $\mu_{0}(n)$ число непоявившихся значений из множества $\aleph$ среди $n$ первых членов последовательности $X$. Эту величину можно интерпретировать как число ячеек, оставшихся пустыми после размещения $n$ частиц в схеме размещения, где $x_{t}-$ номер ячейки, в которую попала $t$-я частица, $1 \leqslant t \leqslant n$.

Асимптотическая нормальность $\mu_{0}(n)$ при $n, N \rightarrow \infty$ имеет место для многих схем независимого размещения частиц (см. [1]). В работах [2,3] была рассмотрена последовательность $X$, образованная $v$-цепочками $x_{t}=\left(y_{t+r_{1}}, \ldots, y_{t+r_{v}}\right)$, построенными по последовательности независимых случайных величин $\left(y_{1}, y_{2}, \ldots\right)$. Были найдены условия, достаточные для асимптотической нормальности $\mu_{0}(n)$ при $n, N \rightarrow \infty$ в случае, когда $n$ и $N^{v}$ имеют одинаковый порядок роста. Задачи о размещении частиц по ячейкам в случае, когда последовательность $X$ является цепью Маркова, исследовались в работе [4]. Асимптотическая нормальность величин, аналогичных $\mu_{0}(n)$, для $m$-зависимых последовательностей при $n, N \rightarrow \infty, n=o\left(N^{v}\right)$, исследовалась в работах $[6,7]$.

В работе получены достаточные условия асимптотической нормальности вектора

$$
\bar{\mu}_{0}=\left(\mu_{0}\left(n_{1}\right), \mu_{0}\left(n_{1}+n_{2}\right), \ldots, \mu_{0}\left(n_{1}+n_{2}+\ldots+n_{r}\right)\right)
$$

в случае, когда величины $N, n_{1}, \ldots, n_{r}$ стремятся к бесконечности одинаково быстро. Одномерные распределения были изучены в работе [5].

* Место работы: Академия криптографии Российской Федерации, e-mail: akrf@rambler.ru

${ }^{\dagger}$ Место работы: Математический институт им. В.А. Стеклова РАН, e-mail: vpchist@mi.ras.ru 


\section{1. Постановка задачи}

Будем предполагать, что последовательность $X$ является стационарной последовательностью $m$-зависимых случайных величин: наборы $\left(x_{s-u}, \ldots, x_{s}\right)$ и $\left(x_{t}, \ldots, x_{t+u}\right)$ независимы при любых целых положительных числах $u, v, s, t, t-s>m$. Введем следующие обозначения:

$$
\begin{gathered}
a_{i}=N \mathbf{P}\left\{x_{t}=i\right\}, \quad t=1,2, \ldots, \quad i \in \aleph, \\
b_{i j}(u)=N^{2} \mathbf{P}\left\{x_{t}=i, x_{t+u}=j\right\}, \quad i, j \in \aleph .
\end{gathered}
$$

Отметим, что

$$
\sum_{i=1}^{N} a_{i}=N, \quad \sum_{i, j=1}^{N} b_{i j}(u)=N^{2}, \quad b_{i j}(u)=a_{i} a_{j},
$$

если $u \geqslant m+1$. Пусть $\chi(C)-$ индикатор события $C$.

Случайную величину

$$
\mu_{0}(n)=\sum_{i=1}^{N} \chi\left(x_{1} \neq i, \ldots, x_{n} \neq i\right),
$$

используя метод включения-исключения, представим в следующем виде (см. [2, 3]):

$$
\mu_{0}(n)=N+\sum_{l=1}^{n} \sum_{t(l) \in T_{l}(n)} \xi_{l}(t(l))
$$

где $t(l)=\left(t_{1}, \ldots, t_{l}\right)$,

$$
\begin{gathered}
T_{l}(n)=\left\{t(l): 1 \leqslant t_{1}<\ldots<t_{l} \leqslant n\right\}, \quad l \geqslant 1, \\
\xi_{l}(t(l))=(-1)^{l} \sum_{i=1}^{N} \chi\left(x_{t_{1}}=\ldots=x_{t_{l}}=i\right) .
\end{gathered}
$$

Цель работы - вывод достаточных условий асимптотической нормальности вектора $\bar{\mu}_{0}$ в схеме серий, в которой

$$
\begin{gathered}
n_{1}=\alpha_{N, 1} N, n_{2}=\alpha_{N, 2} N, \ldots, n_{r}=\alpha_{N, r} N, N \rightarrow \infty, \\
0<\underline{\alpha} \leqslant \alpha_{N, u} \leqslant \bar{\alpha}<\infty, \quad u=1, \ldots, r,
\end{gathered}
$$

а распределение последовательности $X$ меняется так, что при любых $t_{1}<\ldots<t_{l}$ и любых $i_{1}, \ldots, i_{l} \in \aleph$

$$
\begin{gathered}
0<\underline{a} \leqslant a_{i}=N \mathbf{P}\left\{x_{t}=i\right\} \leqslant \bar{a}<\infty \\
0<\underline{c}^{l} \leqslant N^{l} \mathbf{P}\left\{x_{t_{1}}=i_{1}, \ldots, x_{t_{l}}=i_{l}\right\} \leqslant \bar{c}^{l}, \quad l=1,2, \ldots
\end{gathered}
$$

Здесь $\underline{\alpha}, \bar{\alpha}, \underline{a}, \bar{a}, \underline{c}, \bar{c}-$ некоторые постоянные.

Пример $m$-зависимых случайных величин, для которых выполняются условия (8), можно найти в работе [5]. 


\section{2. Асимптотические формулы для моментов}

Асимптотические свойства моментов и асимптотическая нормальность координат вектора $\bar{\mu}_{0}$ изучались в работе [5]. Приведем в обозначениях, удобных для дальнейшего изложения, формулы для $\mathbf{E} \mu_{0}(n)$ и $\mathbf{D} \mu_{0}(n)$, полученные в теореме 1 и следствии 1 работы [5]. Положим $\alpha_{N}=n / N$,

$$
\begin{gathered}
E_{v}\left(\alpha_{N}\right)=\frac{1}{N} \sum_{i=1}^{N} a_{i}^{v} e^{-\alpha_{N} a_{i}}, \quad v=0,1,2, \\
B_{i j}^{(1)}(u)=b_{i i}(u)+b_{i j}(u)+b_{j i}(u)+b_{j j}(u), \\
B_{i j}^{(2)}(u)=b_{i j}(u)+b_{j j}(u), \quad B_{i j}^{(3)}(u)=b_{i j}(u), \\
b_{i j}=\frac{1}{m} \sum_{u=1}^{m} b_{i j}(u), \quad B_{i j}^{(v)}=\frac{1}{m} \sum_{u=1}^{m} B_{i j}^{(v)}(u), \quad v=1,2,3 .
\end{gathered}
$$

Теорема 1. При $n, N \rightarrow \infty u 0<\underline{\alpha} \leqslant \alpha_{N} \leqslant \bar{\alpha}<\infty$ в схеме серий (8) при некотором $0<\delta<1$ выполнены соотношения

$$
\begin{gathered}
\mathbf{E} \mu_{0}(n)=N E_{0}\left(\alpha_{N}\right)-\frac{\alpha_{N}}{2} E_{2}(\alpha)+\frac{\alpha_{N} m}{N} \sum_{i=1}^{N}\left(b_{i i}-a_{i}^{2}\right) e^{-\alpha_{N} a_{i}}+O\left(\frac{1}{N^{\delta}}\right), \\
D \mu_{0}(n)=\sigma^{2} N+O\left(N^{1-\delta}\right),
\end{gathered}
$$

где

$$
\begin{aligned}
& \sigma^{2}=E_{0}\left(\alpha_{N}\right)-E_{0}\left(2 \alpha_{N}\right)-\alpha_{N}\left(E_{1}\left(\alpha_{N}\right)\right)^{2}+ \\
& +2 \alpha_{N} \frac{1}{N^{2}} \sum_{i, j=1}^{N} e^{-\alpha_{N}\left(a_{i}+a_{j}\right)} \sum_{u=1}^{m}\left(b_{i j}(u)-a_{i} a_{j}\right) .
\end{aligned}
$$

В этом разделе мы выведем асимптотическую формулу для $\operatorname{cov}\left(\mu_{0}\left(n_{1}\right), \mu_{0}\left(n_{1}+n_{2}\right)\right)$, необходимую для доказательства теоремы об асимптотической нормальности вектора $\bar{\mu}_{0}$.

Теорема 2. При $n_{1}, n_{2}, N \rightarrow \infty$ в схеме серий (7), (8) для ковариачии величин $\mu_{0}(m), \mu_{0}\left(n_{1}+n_{2}\right)$ при некотором $0<\delta<1$ выполняется равенство

$$
\operatorname{cov}\left(\mu_{0}(m), \mu_{0}\left(n_{1}+n_{2}\right)\right)=N \sigma_{12}+O\left(N^{1-\delta}\right),
$$

¿əe

$$
\sigma_{12}=E_{0}\left(\alpha_{N, 1}+\alpha_{N, 2}\right)-E_{0}\left(2 \alpha_{N, 1}+\alpha_{N, 2}\right)-\alpha_{N, 1} E_{1}\left(\alpha_{N, 1}\right) E_{1}\left(\alpha_{N, 1}+\alpha_{N, 2}\right)+
$$

$+\alpha_{N, 1} m\left[\left(\frac{1}{N^{2}} \sum_{i, j=1}^{N}\left(b_{i j}+b_{j i}\right) e^{-\alpha_{N, 1} a_{i}} e^{-\left(\alpha_{N, 1}+\alpha_{N, 2}\right) a_{j}}\right)-2 E_{1}\left(\alpha_{N, 1}\right) E_{1}\left(\alpha_{N, 1}+\alpha_{N, 2}\right)\right]$,

$$
\alpha_{N, 1}=n_{1} N^{-1}, \alpha_{N, 2}=n_{2} N^{-1}, \text { а функции } E_{0}(\alpha) \text { и } E_{1}(\alpha) \text { определенъь в }(9) \text {. }
$$


Если последовательность $X$ образована независимыми и равномерно распределенными на ふ случайными величинами, то

$$
a_{i}=b_{i j}=1, \quad E_{0}(\alpha)=E_{1}(\alpha)=e^{-\alpha}, \quad i, j=1, \ldots, N
$$

В этом случае согласно теореме 2

$$
\sigma_{12}=e^{-\left(\alpha_{N, 1}+\alpha_{N, 2}\right)}\left(1-\left(1+\alpha_{1}\right) e^{-\alpha_{N, 1}}\right),
$$

что совпадает с формулой (IV. 4.4) в [1].

Используя теоремы 1 и 2, выведем асимптотические формулы для моментов координат вектора

$$
\bar{\mu}_{0}=\left(\varsigma_{1}, \varsigma_{2}, \ldots, \varsigma_{r}\right), \varsigma_{k}=\mu_{0}\left(n_{1}+\ldots+n_{k}\right), 1 \leqslant k \leqslant r .
$$

Положим

$$
n(i)=n_{1}+\ldots+n_{i}, \quad \alpha(i)=\alpha_{N, 1}+\ldots+\alpha_{N, i} .
$$

В схеме серий (7), (8) выполнены соотношения

$$
\begin{gathered}
\mathbf{E}_{\varsigma_{i}}=N E_{0}(\alpha(i))+O(1), \\
\operatorname{cov}\left(\varsigma_{i}, \varsigma_{j}\right)=N \sigma_{i j}+O\left(N^{1-\delta}\right), \quad i, j=1, \ldots, r, \quad 0<\delta<1, \\
\sigma_{i j}=E_{0}(\alpha(j))-E_{0}(\alpha(i)+\alpha(j))-\alpha(i) E_{1}(\alpha(i)) E_{1}(\alpha(j))+ \\
+\alpha(i) m\left[\left(\frac{1}{N^{2}} \sum_{u, v=1}^{N}\left(b_{u v}+b_{v u}\right) e^{-\alpha(i) a_{u}} e^{-(\alpha(j)) a_{v}}\right)-2 E_{1}(\alpha(i)) E_{1}(\alpha(j))\right],
\end{gathered}
$$

$i<j$, где величины $E_{0}(\alpha), E_{1}(\alpha), \alpha(i)$ определены равенствами (9), (13). Эти формулы вытекают из формул теорем 1 и 2 , если заменить в них величины $\alpha_{N}$, $\alpha_{N, 1}, \alpha_{N, 2}$ величинами $\alpha(i), \alpha(i), \alpha(j)-\alpha(i)$ соответственно.

При доказательстве теоремы 2 потребуется ряд вспомогательных утверждений.

Для $(k, l), 0 \leqslant k \leqslant \min \left\{l, n_{1}\right\}, 1 \leqslant l \leqslant n_{1}+n_{2}$, введем множества

$$
T_{l}\left(n_{1}, n_{2}, k\right)=\left\{t(l): 1 \leqslant t_{1}<t_{2}<\ldots<t_{k} \leqslant n_{1}<t_{k+1}<\ldots<t_{l} \leqslant n_{1}+n_{2}\right\} .
$$

Лемма 1. Произведение $\mu_{0}\left(n_{1}\right) \mu_{0}\left(n_{1}+n_{2}\right)$ можно представить в виде

$$
\mu_{0}\left(n_{1}\right) \mu_{0}\left(n_{1}+n_{2}\right)=\mu_{0}\left(n_{1}+n_{2}\right)+D_{n_{1} n_{2}},
$$

где

$$
\begin{gathered}
D_{n_{1} n_{2}}=N(N-1)+\sum_{i \neq j} \sum_{l=1}^{n_{1}+n_{2}}(-1)^{l} \sum_{k=0}^{\min \left\{l, n_{1}\right\}} \sum_{t(l) \in T_{l}\left(n_{1}, n_{2}, k\right)} H(t(l)), \\
H(t(l))=h_{i j}\left(t_{1}\right) \ldots h_{i j}\left(t_{k}\right) h_{j}\left(t_{k+1}\right) \ldots h_{j}\left(t_{l}\right), \\
h_{i j}(t)=\chi\left(x_{t}=i\right)+\chi\left(x_{t}=j\right), \quad i \neq j, \quad h_{j}(t)=\chi\left(x_{t}=j\right) .
\end{gathered}
$$


Доказательство. Используя формулу (3) при $n=n_{1}$ и $n=n_{1}+n_{2}$, получаем:

$$
\begin{gathered}
\mu_{0}\left(n_{1}\right) \mu_{0}\left(n_{1}+n_{2}\right)=\sum_{i, j=1}^{N} \chi\left(x_{1} \neq i, \ldots, x_{n_{1}} \neq i\right) \chi\left(x_{1} \neq j, \ldots, x_{n_{1}+n_{2}} \neq j\right)= \\
=\sum_{i=1}^{N} \prod_{v=1}^{n_{1}+n_{2}}\left(1-h_{i}(v)\right)+\sum_{i \neq j} \prod_{v=1}^{n_{1}}\left(1-h_{i j}(v)\right) \prod_{v=n_{1}+1}^{n_{1}+n_{2}}\left(1-h_{j}(v)\right) .
\end{gathered}
$$

Отсюда следует утверждение леммы, так как

$$
\begin{gathered}
\sum_{i=1}^{N} \prod_{v=1}^{n_{1}+n_{2}}\left(1-h_{i}(v)\right)=\mu_{0}\left(n_{1}+n_{2}\right), \\
\prod_{v=1}^{n_{1}}\left(1-h_{i j}(v)\right) \prod_{v=n_{1}+1}^{n_{1}+n_{2}}\left(1-h_{j}(v)\right)= \\
=1+\sum_{l=1}^{n_{1}+n_{2}}(-1)^{l} \sum_{k=0}^{\min \left\{l, n_{1}\right\}} \sum_{t(l) \in T_{l}\left(n_{1}, n_{2}, k\right)} h_{i j}\left(t_{1}\right) \ldots h_{i j}\left(t_{k}\right) h_{j}\left(t_{k+1}\right) \ldots h_{j}\left(t_{l}\right) .
\end{gathered}
$$

Положим $\tau(t)=(t, t+1, \ldots, t+m)$. Множество $T_{l}\left(n_{1}, n_{2}, k\right)$, определенное равенством (15), разобьем на три части:

$$
T_{l}\left(n_{1}, n_{2}, k\right)=\bigcup_{v=1}^{3} T_{l}^{(v)}\left(n_{1}, n_{2}, k\right),
$$

где

$$
\begin{aligned}
& T_{l}^{(1)}\left(n_{1}, n_{2}, k\right)=\left\{t(l) \in T_{l}\left(n_{1}, n_{2}, k\right): \tau\left(t_{v}\right) \cap \tau\left(t_{u}\right)=\varnothing, u \neq v\right\}, \\
& T_{l}^{(2)}\left(n_{1}, n_{2}, k\right)=\left\{\begin{array}{c}
t(l) \in T_{l}\left(n_{1}, n_{2}, k\right): \text { среди } \tau\left(t_{1}\right), \ldots, \tau\left(t_{l}\right) \\
\text { только одна пара множеств пересекается }
\end{array}\right\}, \\
& T_{l}^{(3)}\left(n_{1}, n_{2}, k\right)=T_{l}\left(n_{1}, n_{2}, k\right) \backslash\left(T_{l}^{(1)}\left(n_{1}, n_{2}, k\right) \cup T_{l}^{(2)}\left(n_{1}, n_{2}, k\right)\right) .
\end{aligned}
$$

Очевидно, что $T_{1}^{(v)}\left(n_{1}, n_{2}, k\right)=\varnothing, v=2,3$.

Используя это разбиение, запишем представление

$$
D_{n_{1}, n_{2}}=D_{n_{1}, n_{2}}^{(1)}+D_{n_{1}, n_{2}}^{(2)}+D_{n_{1}, n_{2}}^{(3)},
$$

где $D_{n_{1}, n_{2}}^{(1)}, D_{n_{1}, n_{2}}^{(2)}$ и $D_{n_{1}, n_{2}}^{(3)}$ определены по формулам, аналогичным (16):

$$
\begin{gathered}
D_{n_{1} n_{2}}^{(1)}=1+\sum_{i \neq j} \sum_{l=1}^{n_{1}+n_{2}}(-1)^{l} \sum_{k=0}^{\min \left\{l, n_{1}\right\}} \sum_{t(l) \in T_{l}^{(1)}\left(n_{1}, n_{2}, k\right)} H(t(l)), \\
D_{n_{1} n_{2}}^{(v)}=\sum_{i \neq j} \sum_{l=2}^{n_{1}+n_{2}}(-1)^{l} \sum_{k=0}^{\min \left\{l, n_{1}\right\}} \sum_{t(l) \in T_{l}^{(v)}\left(n_{1}, n_{2}, k\right)} H(t(l)),
\end{gathered}
$$

a $v=2,3$. 
Лемма 2. В схеме серий (7), (8)

$$
\begin{gathered}
\mathbf{E} D_{n_{1} n_{2}}^{(1)}=N^{2} E_{0}\left(\alpha_{N, 1}\right) E_{0}\left(\alpha_{N, 1}+\alpha_{N, 2}\right)-N E_{0}\left(2 \alpha_{N, 1}+\alpha_{N, 2}\right)+ \\
\quad+\frac{2 m+1}{2} N \alpha_{N, 1} E_{2}\left(\alpha_{N, 1}\right) E_{0}\left(\alpha_{N, 1}+\alpha_{N, 2}\right)+ \\
+\frac{2 m+1}{2} N\left[\left(\alpha_{N, 1}+\alpha_{N, 2}\right) E_{0}\left(\alpha_{N, 1}\right) E_{2}\left(\alpha_{N, 1}+\alpha_{N, 2}\right)+\right. \\
\left.+2 \alpha_{N, 1} E_{1}\left(\alpha_{N, 1}\right) E_{1}\left(\alpha_{N, 1}+\alpha_{N, 2}\right)\right]+O(1)
\end{gathered}
$$

где $E_{v}(\alpha), v=0,1,2$, определены равенствами (9).

Лемма 3. В схеме серий (7), (8)

$$
\mathbf{E} D_{n_{1} n_{2}}^{(2)}=\frac{m}{N} \sum_{i, j=1}^{N}\left(\alpha_{N, 1} B_{i j}^{(1)}+\alpha_{N, 2} B_{i j}^{(3)}\right) e^{-\alpha_{N, 1} a_{i}} e^{-\left(\alpha_{N, 1}+\alpha_{N, 2}\right) a_{j}}+O(1) .
$$

Лемма 4. В схеме серий (7), (8)

$$
\mathbf{E} D_{n_{1} n_{2}}^{(3)}=O(1) .
$$

Отложим на время громоздкие доказательства этих лемм и перейдем к доказательству теоремы 2. Из леммы 1 следует, что

$$
\operatorname{cov}\left(\mu_{0}\left(n_{1}\right), \mu_{0}\left(n_{1}+n_{2}\right)\right)=\mathbf{E} \mu_{0}\left(n_{1}+n_{2}\right)+\mathbf{E} D_{n_{1} n_{2}}-\mathbf{E} \mu_{0}\left(n_{1}\right) \mathbf{E} \mu_{0}\left(n_{1}+n_{2}\right) .
$$

Асимптотические формулы для величин $\mathbf{E} \mu_{0}\left(n_{1}+n_{2}\right)$ и $\mathbf{E} \mu_{0}\left(n_{1}\right)$ получаются из теоремы 1 при $\alpha_{N}=\alpha_{N, 1}+\alpha_{N, 2}$ и $\alpha_{N}=\alpha_{N, 1}$. Кроме этого, согласно равенству (20)

$$
\mathbf{E} D_{n_{1}, n_{2}}=\mathbf{E} D_{n_{1}, n_{2}}^{(1)}+\mathbf{E} D_{n_{1}, n_{2}}^{(2)}+\mathbf{E} D_{n_{1}, n_{2}}^{(3)}
$$

Подставив эти формулы и оценки лемм 2 - 4 в (26), получаем утверждение теоремы 2 .

При доказательстве леммы 2 нам понадобится следующая оценка.

Лемма 5. В схеме серий (7), (8) равномерно по $k, l, 0 \leqslant k \leqslant \min \left\{l, n_{1}\right\}, 2 \leqslant l \leqslant$ $n_{1}+n_{2}$,

$$
\begin{gathered}
\frac{1}{N^{l}}\left|T_{l}^{(1)}\left(n_{1}, n_{2}, k\right)\right|=\frac{\alpha_{N, 1}^{k} \alpha_{N, 2}^{l-k}}{k !(l-k) !}- \\
-\left(m+\frac{1}{2}\right) \frac{1}{N(l-2) !}\left(C_{l-1}^{k-2} \alpha_{N, 1}^{k-1} \alpha_{N, 2}^{l-k}+C_{l-2}^{k} \alpha_{N, 1}^{k} \alpha_{N, 2}^{l-k-1}\right)+ \\
+O\left(\frac{1}{N^{2}}\left(k^{4}+(l-k)^{4}\right) \frac{\alpha_{N, 1}^{k} \alpha_{N, 2}^{l-k}}{k !(l-k) !}\right) .
\end{gathered}
$$

Здесь и далее предполагается, что $C_{b}^{a}=0$ при $a>b$, а также при отрицательных значениях $a$ и/или $b$.

Громоздкое доказательство этой технической леммы мы приведем в конце раздела 2. 
Доказательство леммы 2. При $t(l) \in T_{l}^{(1)}\left(n_{1}, n_{2}, k\right)$ случайные величины $x_{t_{1}}, \ldots, x_{t_{l}}$ независимы. Следовательно,

$$
\mathbf{E} H(t(l))=\left(\frac{a_{i}+a_{j}}{N}\right)^{k}\left(\frac{a_{j}}{N}\right)^{l-k}, \quad i \neq j .
$$

Используя (27) и соотношения $n_{u}=\alpha_{N, u} N, u=1,2$, из (21) выводим

$$
\begin{gathered}
\mathbf{E} D_{n_{1} n_{2}}^{(1)}=N(N-1)-\sum_{i \neq j}\left(\alpha_{N, 1} a_{i}+\left(\alpha_{N, 1}+\alpha_{N, 2}\right) a_{j}\right)+ \\
+\sum_{i \neq j} \sum_{l=2}^{n_{1}+n_{2}}(-1)^{l} S_{l}^{(1)}(i, j),
\end{gathered}
$$

где

$$
\begin{aligned}
& S_{l}^{(1)}(i, j)=\sum_{k=0}^{l} \sum_{t(l) \in T_{l}^{(1)}\left(n_{1}, n_{2}, k\right)} \mathbf{E} H(t(l))= \\
& =\sum_{k=0}^{l}\left(a_{i}+a_{j}\right)^{k} a_{j}^{l-k} \frac{1}{N^{l}}\left|T_{l}^{(1)}\left(n_{1}, n_{2}, k\right)\right|
\end{aligned}
$$

при $2 \leqslant l \leqslant n_{1}$ и

$$
S_{l}^{(1)}(i, j)=\sum_{k=0}^{n_{1}}\left(a_{i}+a_{j}\right)^{k} a_{j}^{l-k} \frac{1}{N^{l}}\left|T_{l}^{(1)}\left(n_{1}, n_{2}, k\right)\right|
$$

при $n_{1}<l \leqslant n_{1}+n_{2}$.

Подставив в эти равенства выражение для $\frac{1}{N^{l}}\left|T_{l}^{(1)}\left(n_{1}, n_{2}, k\right)\right|$, приведенное в лемме 5, получим, что в схеме серий $(7),(8)$

$$
\left|S_{l}^{(1)}(i, j)-\bar{S}_{l}^{(1)}(i, j)\right| \leqslant \frac{c^{l}}{N^{2} l !}, \quad 2 \leqslant l \leqslant n_{1},
$$

где

$$
\begin{gathered}
\bar{S}_{l}^{(1)}(i, j)=\frac{1}{l !}\left(\alpha_{N, 1} a_{i}+\left(\alpha_{N, 1}+\alpha_{N, 2}\right) a_{j}\right)^{l}- \\
-\frac{2 m+1}{2 N}\left(\alpha_{N, 1}\left(a_{i}+a_{j}\right)^{2}+\alpha_{N, 2} a_{j}^{2}\right) \frac{1}{(l-2) !}\left(\alpha_{N, 1} a_{i}+\left(\alpha_{N, 1}+\alpha_{N, 2}\right) a_{j}\right)^{l-2}
\end{gathered}
$$

(эти величины мы определяем для всех $l \geqslant 2$ ), и

$$
\left|S_{l}^{(1)}(i, j)\right| \leqslant \frac{c^{l}}{l !}, \quad\left|\bar{S}_{l}^{(1)}(i, j)\right| \leqslant \frac{c^{l}}{l !}, \quad 2 \leqslant l \leqslant n_{1}+n_{2} .
$$

Здесь $c>0-$ некоторая постоянная.

Из этих соотношений следует, что равномерно по $i, j$

$$
\begin{gathered}
\sum_{l=2}^{n_{1}+n_{2}}(-1)^{l} S_{l}^{(1)}(i, j)=\sum_{l=2}^{n_{1}}(-1)^{l} S_{l}^{(1)}(i, j)+O\left(\frac{1}{N^{2}}\right)= \\
=\sum_{l=2}^{n_{1}}(-1)^{l} \bar{S}_{l}^{(1)}(i, j)+O\left(\frac{1}{N^{2}}\right)=\sum_{l=2}^{\infty}(-1)^{l} \bar{S}_{l}^{(1)}(i, j)+O\left(\frac{1}{N^{2}}\right) .
\end{gathered}
$$

Поэтому из (28) вытекает (23). Лемма 2 доказана. 
Доказательство леммы 3. Из (22) следует, что

$$
\mathbf{E} D_{n_{1} n_{2}}^{(2)}=\sum_{i \neq j} \sum_{l=2}^{n_{1}+n_{2}}(-1)^{l} S_{l}^{(2)}(i, j)
$$

где

$$
S_{l}^{(2)}(i, j)=\sum_{k=0}^{\max \left\{l, n_{1}\right\}} \sum_{t(l) \in T_{l}^{(2)}\left(n_{1}, n_{2}, k\right)} \mathbf{E} H(t(l)) .
$$

Оценим сумму $S_{l}^{(2)}(i, j)$. Положим

$$
\begin{gathered}
T_{l}^{(2)}\left(n_{1}, n_{2}, k, v, u, w\right)= \\
=\left\{t(l) \in T_{l}\left(n_{1}, n_{2}, k\right): t_{v}=w, t_{v+1}=w+u ; t_{f+1}-t_{f} \geqslant m+1, f \neq v\right\},
\end{gathered}
$$

где $1 \leqslant u \leqslant m, 0 \leqslant k \leqslant l, 1 \leqslant v \leqslant l-1,1 \leqslant w \leqslant n_{1}+n_{2}-u$. Если $t(l)$ принадлежит множеству (31), то пара $\left(x_{w}, x_{w+u}\right)$ и набор $\left(x_{t}: t \neq w, w+u\right)$ независимы.

Положим

$$
A_{i j}(w, u)=\left(\left(x_{w}=i\right) \cup\left(x_{w}=j\right)\right) \cap\left(\left(x_{w+u}=i\right) \cup\left(x_{w+u}=j\right)\right) .
$$

Из равенств (1) следует, что

$$
N^{2} \mathbf{P}\left(A_{i j}(w, u)\right)= \begin{cases}B_{i j}^{(1)}(u), & w \leqslant n_{1}, w+u \leqslant n_{1}, \\ B_{i j}^{(2)}(u), & w \leqslant n_{1}, w+u>n_{1}, \\ B_{i j}^{(3)}(u), & w>n_{1},\end{cases}
$$

где величины $B_{i j}^{(f)}(u), f=1,2,3$, определены равенствами (10).

Введем обозначение

$$
G_{l}(k, u, w)=\sum_{v=1}^{l-1}\left|T_{l}^{(2)}\left(n_{1}, n_{2}, k, v, u, w\right)\right| \frac{1}{N^{l}}
$$

Из (32) и равенства (30) следует, что

$$
S_{l}^{(2)}(i, j)=S_{l 1}^{(2)}(i, j)+S_{l 2}^{(2)}(i, j)+S_{l 3}^{(2)}(i, j),
$$

где

$$
\begin{gathered}
S_{l 1}^{(2)}(i, j)=\sum_{k=0}^{\max \left\{l, n_{1}\right\}} \sum_{u=1}^{m}\left(a_{i}+a_{j}\right)^{k-2} a_{j}^{l-k} B_{i j}^{(1)}(u) \sum_{w=1}^{n_{1}-u} G_{l}(k, u, w), \\
S_{l 2}^{(2)}(i, j)=\sum_{k=0}^{\max \left\{l, n_{1}\right\}} \sum_{u=1}^{m}\left(a_{i}+a_{j}\right)^{k-1} a_{j}^{l-k-1} B_{i j}^{(2)}(u) \sum_{w=n_{1}-u+1}^{n_{1}} G_{l}(k, u, w), \\
S_{l 3}^{(2)}(i, j)=\sum_{k=0}^{\max \left\{l, n_{1}\right\}} \sum_{u=1}^{m}\left(a_{i}+a_{j}\right)^{k} a_{j}^{l-k-2} B_{i j}^{(3)}(u) \sum_{w=n_{1}+1}^{n_{1}+n_{2}-u} G_{l}(k, u, w) .
\end{gathered}
$$

Далее нам понадобится следующая оценка. 
Лемма 6. При $N, n_{1}=\alpha_{N, 1} N, n_{2}=\alpha_{N, 2} N \rightarrow \infty, 1 \leqslant u \leqslant m$

$$
G_{l}(k, u, w)=\left\{\begin{array}{lc}
\frac{\alpha_{N, 1}^{k-2} \alpha_{N, 2}^{l-k}}{(k-2) !(l-k) !} \frac{1}{N^{2}}+\nabla_{N}^{(1)}(u), & 1 \leqslant w \leqslant n_{1}-u \\
\nabla_{N}^{(2)}(u), & n_{1}-u<w \leqslant n_{2}, \\
\frac{\alpha_{N, 1}^{k} \alpha_{N, 2}^{l-k}}{k !(l-k-2) !} \frac{1}{N^{2}}+\nabla_{N}^{(3)}(u), & n_{2}<w \leqslant n_{1}+n_{2}-u
\end{array}\right.
$$

где равномерно по $l$ u $k, 0 \leqslant k \leqslant \min \left\{l, n_{1}\right\}, 2 \leqslant l \leqslant n_{1}+n_{2}$,

$$
\nabla_{N}^{(f)}(u)=O\left(\frac{1}{N^{3}} \frac{c^{l} l^{6}}{k !(l-k) !}\right), f=1,3, \quad \nabla_{N}^{(2)}(u)=O\left(\frac{1}{N^{2}} \frac{c^{l} l^{6}}{k !(l-k) !}\right) .
$$

Доказательство этой леммы мы приведем в конце раздела, а сейчас продолжим наши рассуждения.

Используя равенства (35) и лемму 6 , получаем при некотором $c>0$ :

$$
\begin{gathered}
S_{l 1}^{(2)}(i, j)=\sum_{k=0}^{\max \left\{l, n_{1}\right\}} \sum_{u=1}^{m}\left(a_{i}+a_{j}\right)^{k-2} a_{j}^{l-k} B_{i j}^{(1)}(u) \times \\
\times \sum_{w=1}^{n_{1}-u}\left(\frac{\alpha_{N, 1}^{k-2} \alpha_{N, 2}^{l-k}}{(k-2) !(l-k) !} \frac{1}{N^{2}}+O\left(\frac{1}{N^{3}} \frac{c^{l} l^{6}}{k !(l-k) !}\right)\right)= \\
=\frac{m}{N} \sum_{k=0}^{\max \left\{l, n_{1}\right\}}\left(\alpha_{N, 1} \frac{\alpha_{N, 1}^{k-2} \alpha_{N, 2}^{l-k}}{(k-2) !(l-k) !}\left(a_{i}+a_{j}\right)^{k-2} a_{j}^{l-k} B_{i j}^{(1)}+O\left(\frac{1}{N^{2}} \frac{c^{l} l^{6}}{k !(l-k) !}\right)\right)
\end{gathered}
$$

равномерно по $0 \leqslant k \leqslant \max \left\{l, n_{1}\right\}, 2 \leqslant l \leqslant n_{1}+n_{2}$ и по $i, j$. Отсюда выводим, что равномерно по $i, j$

$$
S_{l 1}^{(2)}(i, j)=\frac{\alpha_{N, 1} m}{N} \cdot \frac{\left(\alpha_{N, 1}\left(a_{i}+a_{j}\right)+\alpha_{N, 2} a_{j}\right)^{l-2}}{(l-2) !} B_{i j}^{(1)}+O\left(\frac{c^{l} l^{6}}{N^{2} l !}\right)
$$

при $2 \leqslant l \leqslant n_{1}$ и

$$
\left|S_{l 1}^{(2)}(i, j)\right| \leqslant \frac{c^{l} l^{6}}{l !} \quad \text { при } \quad n_{1}+1 \leqslant l \leqslant n_{1}+n_{2} .
$$

Аналогично находим

$$
S_{l 3}^{(2)}(i, j)=\frac{\alpha_{N, 2} m}{N} \cdot \frac{\left(\alpha_{N, 1}\left(a_{i}+a_{j}\right)+\alpha_{N, 2} a_{j}\right)^{l-2}}{(l-2) !} B_{i j}^{(3)}+O\left(\frac{c^{l} l^{6}}{N^{2} l !}\right)
$$

равномерно по $i, j$,

$$
\begin{gathered}
S_{l 2}^{(2)}(i, j)=O\left(\frac{c^{l} l^{6}}{N^{2} l !}\right), \quad 2 \leqslant l \leqslant n_{1}, \\
\left|S_{l 3}^{(2)}(i, j)\right| \leqslant \frac{c^{l} l^{6}}{l !}, \quad\left|S_{l 2}^{(2)}(i, j)\right| \leqslant \frac{c^{l} l^{6}}{l !}, \quad n_{1}+1 \leqslant l \leqslant n_{1}+n_{2} .
\end{gathered}
$$

Из соотношений (36) - (39) следует, что

$$
\left|S_{l}^{(2)}(i, j)-\bar{S}_{l}^{(2)}(i, j)\right| \leqslant \frac{c^{l}}{N^{2} l !}, \quad 2 \leqslant l \leqslant n_{1},
$$


где

$$
\bar{S}_{l}^{(2)}(i, j)=m \frac{\left(\alpha_{N, 1} a_{i}+\left(\alpha_{N, 1}+\alpha_{N, 2}\right) a_{j}\right)^{l-2}}{N(l-2) !}\left(\alpha_{N, 1} B_{i j}^{(1)}+\alpha_{N, 2} B_{i j}^{(3)}\right)
$$

(эти величины мы определяем для всех $l \geqslant 2$ ), и

$$
\left|S_{l}^{(2)}(i, j)\right| \leqslant \frac{c^{l}}{l !}, \quad\left|\bar{S}_{l}^{(2)}(i, j)\right| \leqslant \frac{c^{l}}{l !}, \quad 2 \leqslant l \leqslant n_{1}+n_{2} .
$$

Здесь $c>0-$ некоторая постоянная.

Из этих соотношений следует, что равномерно по $i, j$

$$
\begin{gathered}
\sum_{l=2}^{n_{1}+n_{2}}(-1)^{l} S_{l}^{(2)}(i, j)=\sum_{l=2}^{n_{1}}(-1)^{l} S_{l}^{(2)}(i, j)+O\left(\frac{1}{N^{2}}\right)= \\
=\sum_{l=2}^{n_{1}}(-1)^{l} \bar{S}_{l}^{(2)}(i, j)+O\left(\frac{1}{N^{2}}\right)=\sum_{l=2}^{\infty}(-1)^{l} \bar{S}_{l}^{(2)}(i, j)+O\left(\frac{1}{N^{2}}\right) .
\end{gathered}
$$

Поэтому из (29) вытекает (24). Лемма 3 доказана.

Доказательство леммы 4. Согласно (22)

$$
\mathbf{E} D_{n_{1} n_{2}}^{(3)}=\sum_{i \neq j} \sum_{l=2}^{n_{1}+n_{2}}(-1)^{l} S_{l}^{(3)}(i, j)
$$

где

$$
S_{l}^{(3)}(i, j)=\sum_{k=0}^{\max \left\{l, n_{1}\right\}} \sum_{t(l) \in T_{l}^{(3)}\left(n_{1}+n_{2}, k\right)} \mathbf{E} H(t(l)),
$$

величины $H(t(l)$ определены равенствами (21). Из условий (8) следует, что слагаемые этой суммы не превосходят $(c / N)^{l}$, где $c>0-$ некоторая постоянная. Таким образом,

$$
S_{l}^{(3)}(i, j) \leqslant\left(\frac{c}{N}\right)^{l}\left|T_{l}^{(3)}\left(n_{1}, n_{2}\right)\right|,
$$

где $T_{l}^{(3)}\left(n_{1}, n_{2}\right)=\bigcup_{k=0}^{\max \left\{l, n_{1}\right\}} T_{l}^{(3)}\left(n_{1}, n_{2}, k\right)$.

Оценим мощность этого множества следующим образом. Выберем $l-2$ координат вектора $t(l)$ (это можно сделать не более чем $C_{n_{1}+n_{2}}^{l-2}$ способами). Из этих координат выберем $t_{i}, t_{j}$ и добавим к ним $t_{i}+u_{1}, t_{j}+u_{2}, 1 \leqslant u_{1} \leqslant m, 1 \leqslant u_{2} \leqslant m\left(\right.$ всего $m^{2} C_{l-2}^{2}$ вариантов). Следовательно,

$$
\left|T_{l}^{(3)}\left(n_{1}, n_{2}\right)\right| \leqslant m^{2} C_{l-2}^{2} C_{n_{1}+n_{2}}^{l-2} \leqslant m^{2} N^{l-2} \frac{\left(\alpha_{N, 1}+\alpha_{N, 2}\right)^{l-2}}{(l-2) !} l^{2} .
$$

Отсюда и из (41) получаем:

$$
\left|S_{l}^{(3)}(i, j)\right| \leqslant \frac{l^{2} c_{1}^{l}}{(l-2) ! N^{2}}
$$

где $c_{1}>0$ - некоторая постоянная. Подставим (42) в (40), и учтем, что внешняя сумма в $(40)$ имеет $N(N-1)$ слагаемых, а ряд $\sum_{l=2}^{\infty} l^{2} c_{1}^{l-2} /((l-2) !)$ сходится. Получим (25). Лемма 4 доказана. 
Наконец, вернемся к леммам 5 и 6 . Для первой из них понадобятся новые вспомогательные утверждения. Положим

$$
\begin{gathered}
M_{v}^{(u)}(k, l)=\left\{t(l) \in T_{l}\left(n_{1}, n_{2}, k\right): t_{v+1}-t_{v}=u\right\}, 1 \leqslant u \leqslant m, \\
M_{v}(k, l)=\bigcup_{u=1}^{m} M_{v}^{(u)}(k, l), \quad M_{v}^{(u, w)}(k, l)=\left\{t(l) \in M_{v}^{(u)}(k, l): t_{v}=w\right\} .
\end{gathered}
$$

Лемма 7. При $u \in\{1, \ldots, m\}, 0 \leqslant k \leqslant \min \left\{l, n_{1}\right\}, 2 \leqslant l \leqslant n_{1}+n_{2}$, справедливо равенство

$$
\sum_{v=1}^{l-1}\left|M_{v}^{(u)}(k, l)\right|=\left(n_{1}-u\right) C_{n_{2}}^{l-k} C_{n_{1}-u-1}^{k-2}+u C_{n_{1}+n_{2}-u-1}^{l-2}+\left(n_{2}-u\right) C_{n_{1}}^{k} C_{n_{2}-u-1}^{l-k-2} .
$$

Доказательство. Найдем $\left|M_{v}^{(u, w)}(k, l)\right|$. Рассмотрим три случая.

1) $w \leqslant n_{1}-u$. В этом случае определены $t_{v}=w, t_{v+1}=w+u$; числа $t_{1}, \ldots, t_{v-1}$ нужно выбрать из $\{1,2, \ldots, w-1\}$. Это можно сделать $C_{w-1}^{v-1}$ способами. Цепочку $t_{v+2}, \ldots, t_{k}$ из $\left\{w+n+1, \ldots, n_{1}\right\}$ можно выбрать $C_{n_{1}-w-u}^{k-v-1}$ способами и цепочку $t_{k+1}, \ldots, t_{l}$ из $\left\{n_{1}+1, \ldots, n_{1}+n_{2}\right\}$ можно выбрать $C_{n_{2}}^{l-k}$ способами. Таким образом,

$$
\left|M_{v}^{(u, w)}(k, l)\right|=C_{w-1}^{v-1} C_{n_{1}-w-u}^{k-v-1} C_{n_{2}}^{l-k}, \quad 1 \leqslant w \leqslant n_{1}-u .
$$

2) $n_{1}-u<w \leqslant n_{1}$. В этом случае

$$
\left|M_{v}^{(u, w)}(k, l)\right|=C_{w-1}^{v-1} C_{n_{1}+n_{2}-w-u}^{l-v-1} .
$$

3) $n_{1}<w \leqslant n_{1}+n_{2}-u$. В этом случае

$$
\left|M_{v}^{(u, w)}(k, l)\right|=C_{n_{1}}^{k} C_{w-n_{1}-1}^{v-k-1} C_{n_{1}+n_{2}-w-u}^{l-v-1} .
$$

Используя формулы (45) - (47) для вычисления величины

$$
\sum_{w}^{(u)}(k, l)=\sum_{v=1}^{l-1}\left|M_{v}^{(u, w)}(k, l)\right|
$$

приходим к равенствам

$$
\sum_{w}^{(u)}(k, l)= \begin{cases}C_{n_{2}}^{l-k} C_{n_{1}-u-1}^{k-2}, & 1 \leqslant w \leqslant n_{1}-u \\ C_{n_{1}+n_{2}-u-1}^{l+}, & n_{1}-u<w \leqslant n_{1} \\ C_{n_{1}}^{k} C_{n_{2}-u-1}^{l-2}, & n_{1}<w \leqslant n_{1}+n_{2}-u\end{cases}
$$

Суммируя (49) по $w$, получаем утверждение леммы 7.

Лемма 8. При $0 \leqslant k \leqslant \min \left\{l, n_{1}\right\}, 2 \leqslant l \leqslant n_{1}+n_{2}$

$$
\begin{gathered}
\sum_{v=1}^{l-1}\left|M_{v}(k, l)\right|=(k-1) C_{n_{2}}^{l-k}\left(C_{n_{1}}^{k}-C_{n_{1}-m}^{k}\right)+ \\
+(l-k-1) C_{n_{1}}^{k}\left(C_{n_{2}}^{l-k}-C_{n_{2}-m}^{l-k}\right)+\Delta(k, l), \\
|\Delta(k, l)| \leqslant m \frac{\left(n_{1}+n_{2}-2\right)^{l-2}}{(l-2) !} .
\end{gathered}
$$


Доказательство. Просуммируем по $u \in\{1, \ldots, m\}$ первые два слагаемых правой части равенства (44), используя при этом равенство

$$
\sum_{u=1}^{m}\left(n_{1}-u\right) C_{n_{1}-u-1}^{k-2}=(k-1) \sum_{u=1}^{m} C_{n_{1}-u}^{k-1}=(k-1)\left(C_{n_{1}}^{k}-C_{n_{1}-m}^{k}\right)
$$

и оценку

$$
\sum_{u=1}^{m} u C_{n_{1}+n_{2}-u-1}^{l-2} \leqslant m \frac{\left(n_{1}+n_{2}-2\right)^{l-2}}{(l-2) !} .
$$

Аналогично суммируется третье слагаемое. Отсюда, учитывая, что

$$
\sum_{u=1}^{m} \sum_{v=1}^{l-1}\left|M_{v}^{(u)}(k, l)\right|=\sum_{v=1}^{l-1}\left|M_{v}(k, l)\right|,
$$

получаем утверждение леммы.

Далее нам понадобятся вытекающие из определений оценки

$$
0 \leqslant \sum_{v=1}^{l-1}\left|M_{v}(k, l)\right|-\left|\bigcup_{v=1}^{l-1} M_{v}(k, l)\right| \leqslant \sum_{1 \leqslant v_{1}<v_{2} \leqslant l-1}\left|M_{v_{1}}(k, l) \cap M_{v_{2}}(k, l)\right|,
$$

$l \geqslant 2$, и равенства

$$
\begin{gathered}
\left|T_{1}^{(1)}\left(n_{1}, n_{2}, k\right)\right|=\left|T_{1}\left(n_{1}, n_{2}, k\right)\right|, \quad k \in\{0,1\}, \\
\left|T_{l}^{(1)}\left(n_{1}, n_{2}, k\right)\right|=\left|T_{l}\left(n_{1}, n_{2}, k\right)\right|-\left|\bigcup_{v=1}^{l-1} M_{v}(k, l)\right|, \quad l \geqslant 2 .
\end{gathered}
$$

Оценим правую часть в (50).

Лемма 9. При $0 \leqslant k \leqslant \min \left\{l, n_{1}\right\}, 2 \leqslant l \leqslant n_{1}+n_{2}$

$$
\begin{gathered}
\sum_{1 \leqslant v_{1}<v_{2} \leqslant l-1}\left|M_{v_{1}}(k, l) \cap M_{v_{2}}(k, l)\right| \leqslant \\
\leqslant l^{2} m^{2}\left(C_{n_{1}}^{k-2} C_{n_{2}}^{l-k}+C_{n_{1}}^{k-1} C_{n_{2}}^{l-k-1}+C_{n_{1}}^{k} C_{n_{2}}^{l-k-2}\right) .
\end{gathered}
$$

Доказательство. Пусть $t_{v_{1}+1}=t_{v_{1}}+u_{1} \leqslant n_{1}, t_{v_{2}+1}=t_{v_{2}}+u_{2} \leqslant n_{1}$ и $v_{1}<v_{2}$. В этом случае при заданных $u_{1}$ и $u_{2}$ нужно выбирать лишь $l-2$ координаты вектора $t(l)=$ $\left(t_{1}, \ldots, t_{l}\right)$, так как $t_{v_{1}+1}, t_{v_{2}+1}$ уже определены. Число способов такого выбора не превосходит $C_{n_{1}}^{k-2} C_{n_{2}}^{l-k}$. Пары $\left(u_{1}, u_{2}\right)$ выбираются не более чем $m^{2}$ способами. Таким образом,

$$
\left|M_{v_{1}}(k, l) \cap M_{v_{2}}(k, l)\right| \leqslant m^{2} C_{n_{1}}^{k-2} C_{n_{2}}^{l-k},
$$

где $t_{v_{1}+1} \leqslant n_{1}, t_{v_{2}+1} \leqslant n_{1}$.

Аналогично оцениваются оставшиеся два случая взаимного расположения $t_{v_{1}+1}$, $t_{v_{2}+1}$ :

$$
\begin{gathered}
\left|M_{v_{1}}(k, l) \cap M_{v_{2}}(k, l)\right| \leqslant m^{2} C_{n_{1}}^{k-1} C_{n_{2}}^{l-k-1}, \quad t_{v_{1}+1} \leqslant n_{1}, t_{v_{2}+1}>n_{1}, \\
\left|M_{v_{1}}(k, l) \cap M_{v_{2}}(k, l)\right| \leqslant m^{2} C_{n_{1}}^{k} C_{n_{2}}^{l-k-2}, \quad t_{v_{1}+1}>n_{1}, t_{v_{2}+1}>n_{1} .
\end{gathered}
$$

Из полученных оценок следует утверждение леммы 9. 
Доказательство леммы 5. Используя формулы (50) и (51), равенство

$$
\left|T_{l}\left(n_{1}, n_{2}, k\right)\right|=C_{n_{1}}^{k} C_{n_{2}}^{l-k}, \quad \text { где } \quad n_{1}=\alpha_{N, 1} N, n_{2}=\alpha_{N, 2} N,
$$

леммы 8 и 9 , получаем утверждение леммы 5 .

Доказательство леммы 6. Из равенства

$$
T_{l}^{(2)}\left(n_{1}, n_{2}, k, v, u, w\right)=M_{v}^{(u, w)}(k, l) \backslash \bigcup_{f \neq v}\left(M_{f}(k, l) \cap M_{v}^{(u, w)}(k, l)\right) .
$$

где $M_{f}(k, l), M_{v}^{(u, w)}(k, l)$ определены равенствами (43), следует, что

$$
\begin{gathered}
0 \leqslant\left|T_{l}^{(2)}\left(n_{1}, n_{2}, k, v, u, w\right)\right|-\left|M_{v}^{(u, v)}(k, l)\right| \leqslant \\
\leqslant \sum_{f \neq l}\left|M_{v}^{(u, w)}(k, l) \cap M_{f}(k, l)\right| .
\end{gathered}
$$

Оценим выражение в правой части (52). Пусть

$$
n_{1}=\alpha_{N, 1} N, \quad n_{2}=\alpha_{N, 2} N, \quad t_{v+1}=t_{v}+u, t_{f+1}-t_{f} \leqslant m, t_{v}=w .
$$

Рассмотрим сначала случай, когда $t_{v}, t_{v+1}, t_{f}, t_{f+1}$ не превосходят $n_{1}$. Величины $t_{v}, t_{v+1}$ определены однозначно; величины $t_{f}, t_{f+1}$ можно выбрать не более, чем $m n_{1}$ способами. Оставшиеся координаты вектора $t(l)$ при заданном расположении $t_{v}, t_{v+1}, t_{f}, t_{f+1}$ можно выбрать $C_{n_{1}}^{k-4} C_{n_{2}}^{l-k}$ способами. Таким образом, в этом случае сумма соответствующей части слагаемых суммы (52) не превосходит

$$
(l-1) m n_{1} C_{n_{1}}^{k-4} C_{n_{2}}^{l-k} \leqslant \frac{m(l-1) \alpha_{N, 1} \alpha_{N, 1}^{k-u} \alpha_{N, 2}^{l-k}}{(k-4) !(l-k) !} \leqslant \frac{l^{5} c_{1}^{l}}{k !(l-k) !} N^{l-3},
$$

где $c_{1}>0-$ некоторая постоянная.

Аналогично рассматриваются остальные варианты расположения $t_{v}, t_{v+1}, t_{f}$, $t_{f+1}$. Для их числа справедливы такие же оценки. Объединив эти оценки, получим при некотором $c>c_{1}$ неравенство

$$
\sum_{f \neq v}\left|M_{v}^{(u, w)}(k, l) \cap M_{f}(k, l)\right| \leqslant \frac{l^{5} c^{l}}{k !(l-k) !} N^{l-3}, \quad 1 \leqslant u \leqslant m .
$$

Из $(33)$ - (53) следует, что равномерно по $l$ и $k, 0 \leqslant k \leqslant \min \left\{l, n_{1}\right\}, 2 \leqslant l \leqslant n_{1}+n_{2}$,

$$
G_{l}(k, u, w)=\frac{1}{N^{l}} \sum_{w}^{(u)}(k, l)+O\left(\frac{1}{N^{3}} \frac{c^{l} l^{6}}{k !(l-k) !}\right) .
$$

Используя (49), получаем:

$$
\begin{gathered}
\sum_{w}^{(u)}(k, l)=\left\{\begin{array}{c}
\frac{\alpha_{N, 1}^{k-2} \alpha_{N, 2}^{l-k}}{(k-2) !(l-k) !} N^{l-2}\left(1+O\left(\frac{c^{l} k^{2}}{N}\right)\right), \quad 1 \leqslant w \leqslant n_{1}-u, \\
\frac{\alpha_{N, 1}^{k} \alpha_{N, 2}^{l-k-2}}{k !(l-k-2) !} N^{l-2}\left(1+O\left(\frac{(l-k)^{2}}{N}\right)\right), n_{2}<w \leqslant n_{1}+n_{2}-u,
\end{array}\right. \\
\sum_{w}^{(u)}(k, l) \leqslant \frac{\left(\alpha_{N, 1}+\alpha_{N, 2}\right)^{l-2}}{(l-2) !} N^{l-2}, \quad n_{1}-u<w \leqslant n_{2} .
\end{gathered}
$$

Из этих оценок следует утверждение леммы 6. 


\section{3. Асимптотическая нормальность}

Для проверки асимптотической нормальности вектора $\bar{\mu}_{0}$ воспользуемся приемом Крамера - Уолда, согласно которому для доказательства сходимости по распределению случайных векторов достаточно доказать сходимость по распределению всевозможных линейных комбинаций их координат (см. [8], с. 76).

Положим

$$
Z_{N}=c_{1} \varsigma_{1}+c_{2} \varsigma_{2}+\ldots+c_{r} \varsigma_{r}, \quad \varsigma_{k}=\mu_{0}(n(k)), \quad 1 \leqslant k \leqslant r .
$$

При доказательстве асимптотической нормальности этой величины воспользуемся теоремой 2 работы [5]. Эта теорема является незначительной переформулировкой теоремы 1 работы [9], обобщающей теорему 2 работы [10].

Величину $\varsigma_{k}$ представим в виде

$$
\varsigma_{k}=\sum_{i=1}^{N}\left(1-H_{i}^{(k)}(1)\right) \cdots\left(1-H_{i}^{(k)}(n(r))\right),
$$

где

$$
\begin{gathered}
H_{i}^{(k)}(t)= \begin{cases}h_{i}(t), & 1 \leqslant t \leqslant n(k), \\
e_{i}(t), & n(k)<t \leqslant n(r),\end{cases} \\
e_{i}(t)=0, i=1, \ldots, N, \quad 1 \leqslant t \leqslant n(r), \quad h_{i}(t)=\chi\left(x_{t}=i\right) .
\end{gathered}
$$

Отсюда получим следующее выражение:

$$
\varsigma_{k}=N+\sum_{i=1}^{N} \sum_{l=1}^{n(r)}(-1)^{l} \sum_{t(l) \in T_{l}(n(r))} H_{i}^{(k)}\left(t_{1}\right) \cdots H_{i}^{(k)}\left(t_{l}\right), \quad k=1, \ldots, r,
$$

где множество $T_{l}(n)$ определено равенством (5). Положим

$$
\begin{aligned}
\xi_{l}^{(k)}(t(l))= & (-1)^{l} \sum_{i=1}^{N} H_{i}^{(k)}\left(t_{1}\right) \cdots H_{i}^{(k)}\left(t_{l}\right), \quad k=1, \ldots, r, \\
& \xi_{l}(t(l))=\sum_{k=1}^{r} c_{k} \xi_{l}^{(k)}(t(l)), \quad l \geqslant 1 .
\end{aligned}
$$

Используя эти обозначения и равенства $(54),(55)$, запишем $Z_{N}$ в виде

$$
Z_{N}=N \sum_{k=1}^{r} c_{k}+\sum_{l=1}^{n(r)} \sum_{t(l) \in T_{l}(n(r))} \xi_{l}\left(t_{l}\right),
$$

аналогичном виду $\mu_{0}(n, N)$, приведенному в формуле (4) работы [5]. Представим множество $T_{l}(n)$ в виде объединения трех не пересекающихся множеств

$$
T_{l}(n)=T_{l}^{(1)}(n) \cup T_{l}^{(2)}(n) \cup T_{l}^{(3)}(n),
$$

где

$$
\begin{gathered}
T_{l}^{(1)}(n)=\left\{t(l): t(l) \in T_{l}(n), \tau\left(t_{i}\right) \cap \tau\left(t_{j}\right)=\varnothing, i \neq j\right\}, \\
T_{l}^{(2)}(n)=\left\{\begin{array}{l}
t(l): t(l) \in T_{l}(n), \text { среди } \tau\left(t_{1}\right), \ldots, \tau\left(t_{l}\right) \\
\text { только одна пара множеств пересекается }
\end{array}\right\},
\end{gathered}
$$




$$
T_{l}^{(3)}(n)=T_{l}(n) \backslash\left(T_{l}^{(1)}(n) \cup T_{l}^{(2)}(n)\right) .
$$

Положим

$$
\begin{gathered}
Z_{N}^{(v)}=\chi(v=1) N \sum_{k=1}^{r} c_{k}+\sum_{l=1}^{\phi-1} \sum_{t(l) \in T_{l}^{(v)}(n(r))} \xi_{l}(t(l)), \quad v=1,2,3, \\
\Delta_{N}=Z_{N}^{(2)}+Z_{N}^{(3)} .
\end{gathered}
$$

Тогда $Z_{N}=Z_{N}^{(1)}+\Delta_{N}$. Аналогично рассуждениям работ $[3,5]$ проверяется, что $\Delta_{N} / \sqrt{N}$ стремится по вероятности к нулю при $N \rightarrow \infty$ в схеме серий (7), (8). В свою очередь, к $Z_{N}^{(1)}$ при $\left(c_{1}, \ldots, c_{r}\right) \neq(0, \ldots, 0)$ применяется теорема 2 работы [5]. Проверка выполнения условий этой теоремы в схеме серий (7), (8) для величины $Z_{N}^{(1)}$ проводится так же как в [3, 5].

Эти рассуждения вместе с приемом Крамера - Уолда приводят к следующему утверждению.

Теорема 3. Если в схеме серий (7), (8) при $N \rightarrow \infty$ определитель $d_{r}=\operatorname{det}\left(\sigma_{i j}\right)$ ковариационной матрицы $\left(\sigma_{i j}\right)$ отделен от нуля $\left(d_{r} \geqslant \varepsilon>0\right)$, то распределение векmopa

$$
\begin{gathered}
\frac{1}{\sqrt{N}}\left(\mu_{0}(n)-N E_{1}\left(\alpha_{N, 1}\right), \mu_{0}\left(n_{1}+n_{2}\right)-\right. \\
\left.-N E_{1}\left(\alpha_{N, 1}+\alpha_{N, 2}\right), \ldots, \mu_{0}\left(n_{1}+\ldots+n_{r}\right)-N E_{1}\left(\alpha_{N, 1}+\ldots+\alpha_{N, r}\right)\right)
\end{gathered}
$$

сходится $к$ невырожденному $r$-мерному нормальному распределению с нулевым вектором математических ожиданий и ковариационной матрицей $\left(\sigma_{i j}\right)$, определенной равенствами (14).

Замечание 1. Используя (2), (8), (13) и равенства

$$
\frac{1}{N^{2}} \sum_{u, v=1}^{N} b_{u v} a_{u}=\frac{1}{N^{2}} \sum_{u, v=1}^{N} b_{u v} a_{v}=a^{2}, \quad a^{2}=\frac{1}{N} \sum_{k=1}^{N} a_{k}^{2}
$$

при $\bar{\alpha} \rightarrow 0, \underline{\alpha} / \bar{\alpha} \geqslant \varepsilon>0$, получаем:

$$
\sigma_{i j}=\frac{1}{2} \alpha^{2}(i) a^{2}+O\left(\bar{\alpha}^{3}\right)
$$

Используя это равенство, вычислим определитель матрицы $\left(\sigma_{i j}\right)$. Для этого вынесем из матрицы постоянный множитель $\frac{1}{2} a^{2}$ и вычтем первый столбец из второго. Затем вычтем второй столбец из третьего и т.д. В итоге в матрице все элементы, стоящие выше главной диагонали, станут равны нулю. Перемножив элементы, находящиеся на главной диагонали, получим, что

$$
\begin{gathered}
\operatorname{det}\left(\sigma_{i j}\right)=\frac{1}{2^{r}} a^{2 r} \alpha_{N, 1}^{2} \alpha_{N, 2} \ldots \alpha_{N, r} \times \\
\times(\alpha(1)+\alpha(2))(\alpha(2)+\alpha(3)) \ldots(\alpha(r-1)+\alpha(r))(1+O(\bar{\alpha})) .
\end{gathered}
$$

Из (56) следует, что условие положительности определителя в теореме 3 выполнено, по крайней мере, при достаточно малых значениях $\bar{\alpha}$.

Авторы благодарны В. Г. Михайлову за интерес к работе и полезные замечания. 


\section{Список литературы}

1. Колчин В. Ф., Севастьянов Б. А., Чистяков В. П., Случайные размещения, М.: Наука, 1976.

2. Тихомирова М. И., Чистяков В. П., "Об асимптотике моментов числа непоявившихся s-цепочек", Дискретная математика, 9:1 (1997), 12-29.

3. Тихомирова М. И., "Асимптотическая нормальность числа непоявившихся несплошных цепочек исходов независимых испытаний”, Дискретная математика, 21:2 (2009), $112-125$.

4. Зубков А. М., "Цепи Маркова, близкие к последовательности независимых испытаний”, Математические заметки, 25:3 (1979), 465-474.

5. Тихомирова М. И., Чистяков В. П., “Асимптотическая нормальность числа непоявившихся значений m-зависимых случайных величин", Математические вопросы криптографии, 2:1 (2011), 119-129.

6. Михайлов В. Г., “Асимптотическая нормальность в схеме конечно-зависимого размещения частиц по ячейкам", Математический сборник, 119(161):4(12) (1982), 509-520.

7. Шойтов А. М., "Нормальное приближение в задачах об эквивалентных цепочках", $B$ сб.: Труды по дискретной математике, 10, М.: ФИЗМАТЛИТ, 2007, 326-349.

8. Биллингсли П., Сходимость вероятностных мер, М.: Наука, 1977.

9. Михайлов В. Г., "Об одной теореме Янсона", Теория вероятн. и ее примен, 36 (1991), $168-170$.

10. Janson S., "Normal convergence by higher semi invariants with applications to sums dependent random variables and random graphs", Ann. Probab., 16:1 (1988), 305-312.

Статья поступила 11.12.2013. 